\title{
Experimental setup to detect superconducting wire motion
}

\author{
K. Ruwali \\ The Graduate University for Advanced Studies, 1-1 Oho, Tsukuba, Ibaraki, 305, Japan \\ A. Yamanaka and Y. Teramoto \\ Research Center, Toyobo Co. Ltd., 2-1-1, Katata, Ohtsu, Shiga 520-0292, Japan \\ K. Nakanishi and K. Hosoyama \\ KEK High Energy Accelerator Research Organization, 1-1 Oho, Tsukuba, Ibaraki, 305-0801, Japan
}

(Received 11 September 2008; published 21 April 2009)

\begin{abstract}
An experimental setup was designed and fabricated to study superconducting wire motion under the influence of electromagnetic force. Experiments were conducted at $4.2 \mathrm{~K}$ by varying the experimental conditions such as the tension to the superconducting wire and different insulating materials at the interface of the superconducting wire and head part. The insulating materials used in the experiments were polyimide film and a high strength polyethylene fiber cloth, Dyneema. Details of the experimental setup and the test results are reported in this paper.
\end{abstract}

DOI: 10.1103/PhysRevSTAB.12.042401

PACS numbers: 07.05.Fb, 07.55.Db, 81.05.Lg, 84.71.Ba

\section{INTRODUCTION}

Mechanical disturbances, such as wire motion, caused by the Lorentz force in magnet coils are the dominant source of performance degradation and thus a major concern in the design of large high field magnets [1]. Frictional heat generated by wire motion is one of the main causes of training in superconducting magnets [2]. The voltage induced by wire motion was measured in the TRISTAN insertion quadrupole magnet [3]. Hence, frictional properties of the wire and the winding structure are important parameters for characterizing the stability of superconducting windings. Kensley and Iwasa [4] made a detailed study of sliding behavior at $4.2,77$, and $295 \mathrm{~K}$ for a number of polymers, laminated composites, and coated pieces sliding against either copper or aluminum.

The experiments on superconducting wire motion [5] in a magnetic field were carried out using small coils wound on stainless steel bobbins with polyimide film as the insulating material and also with high strength polyethylene fiber (Dyneema: DF) reinforced plastic (DFRP) bobbins. The Dyneema fiber has negative thermal expansion [6] and a low coefficient of friction [7]. It was found that voltage spikes generated due to sudden wire motion in the case of the DFRP bobbin were few and small in amplitude. The speculations are as follows: the negative thermal expansion of the DFRP bobbin restrains the wire motion and the low coefficient of friction between superconducting wire and DFRP bobbin reduces sudden motions.

In order to study the effect of the frictional coefficient of insulating material on superconducting wire motion under the influence of electromagnetic force, a special experimental setup was designed and fabricated. The main distinctiveness of the experimental setup is that the tension of the superconducting wire can be changed during the experiments.

In this work, two different types of insulating material were used to study superconducting wire motion under electromagnetic force. They were polyimide film (125 $\mu \mathrm{m}$ thick) and high strength polyethylene fiber cloth sheet Dyneema ${ }^{\circledR}$ SK-60 made by Toyobo Co. Ltd., Japan. Dyneema cloth is a plain wave having 15 yarns/inch with $165 \mathrm{~g} / \mathrm{m}^{2}$.

\section{EXPERIMENTAL SETUP}

The sample holder shown in Fig. 1(a) consists of two parts, a semicircular head of radius $18 \mathrm{~mm}$ and a body part fabricated using G-10. The superconducting wire is wound on the head part and soldered to the copper terminals. The head part is installed and fixed to the superconducting solenoid using support bars. The body part can move upward to change the tension of superconducting wire. Insulating material is inserted between the head part and superconducting wire.

The current is fed to the superconducting wire using an external power supply and the solenoid provides the magnetic field. Voltage taps to measure the signal due to the superconducting wire motion are connected at the end of the head part. To reduce the voltage tap loop area, a groove was incorporated in the head part and the voltage tap wire was passed through it. The voltage tap signal is measured by a pen recorder [8] or a 16-bit data recorder with a sampling rate of $1 \mathrm{MS} / \mathrm{s}$. Sudden wire motion was indicated by observing the voltage spikes.

In order to apply the tension to the superconducting wire, a stainless wire is attached to the body part of the sample holder from the top flange and tension is applied by 


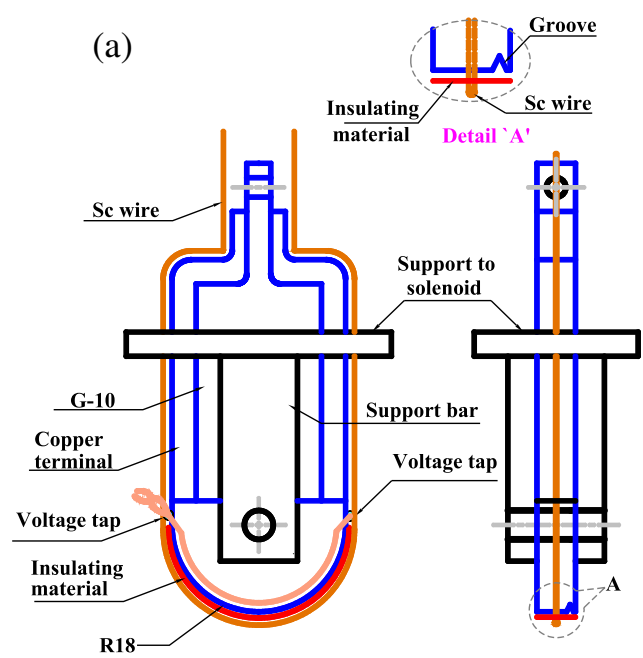

(b)

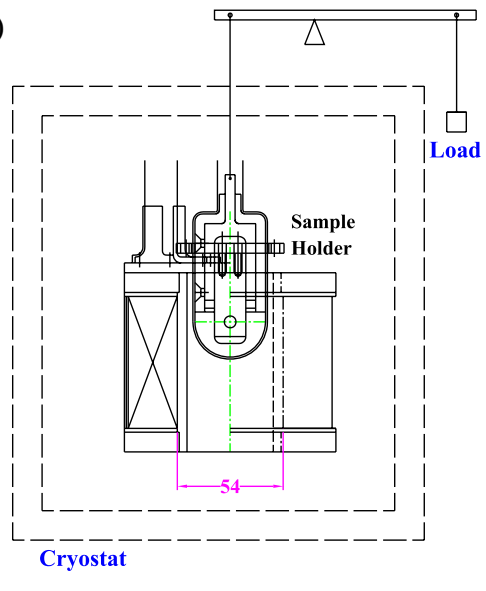

FIG. 1. (Color) (a) Schematic view of sample holder. (b) Schematic view of experimental setup.

a lever mechanism. The tension can be changed as required by adjusting the load. A schematic view of the experimental setup is shown in Fig. 1(b).

The superconducting wire is set on the head part and the tension, $T$, provides a thrust force $F_{t}$ (of the superconducting wire) against the head part. The relation between the thrust force per unit length and tension is

$$
F_{t}=T / r,
$$

where $r=$ radius of the semicircular head.

\section{EXPERIMENT METHODOLOGY AND FINDINGS}

\section{A. Experiment procedure}

The superconducting wire was made of NbTi filaments in a $\mathrm{Cu}$ matrix with formvar insulation. The copper-tosuperconductor area ratio is 1.8 and the filament diameter

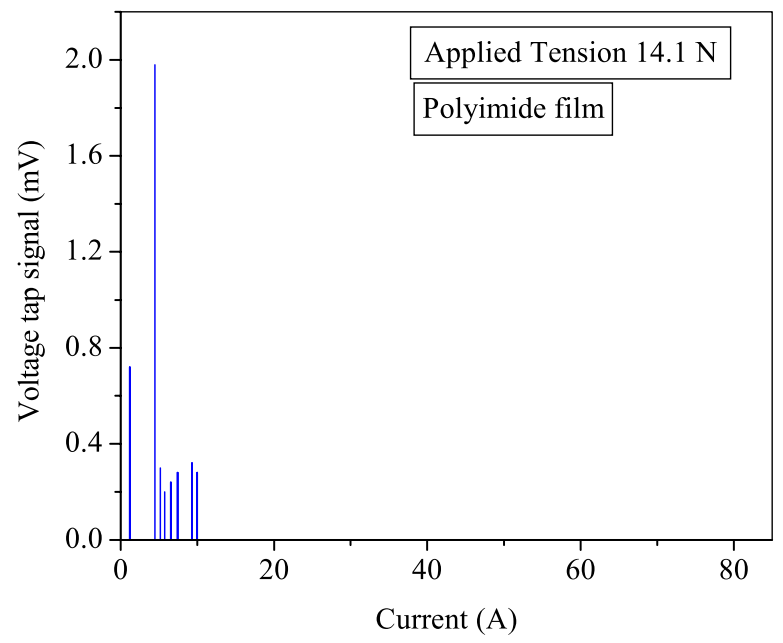

FIG. 2. (Color) Voltage tap signal as a function of current. is $6 \mu \mathrm{m}$. The short sample critical current of the wire is $640 \mathrm{~A}$ in zero field and $260 \mathrm{~A}$ in a field of $6 \mathrm{~T}$.

Measurements were carried out at $4.2 \mathrm{~K}$ using polyimide film and Dyneema cloth as an insulating material between the head part and superconducting wire. During all experiments, a constant magnetic field of $6 \mathrm{~T}$ was applied by solenoid. The current in the superconducting wire was ramped from 0 to $128 \mathrm{~A}$. The current ramp up and ramp down rate was $0.85 \mathrm{~A} / \mathrm{s}$ with a flattop time of $60 \mathrm{~s}$. In order to examine the effect of the current ramp rate on the superconducting wire motion, the current ramp rate was changed from 0.4 to $0.85 \mathrm{~A} / \mathrm{s}$. The superconducting wire tension was varied from 7.1 to $31.8 \mathrm{~N}$ to study the effect of tension on the superconducting wire motion. Experiments were performed with the same conditions for both base insulating materials.
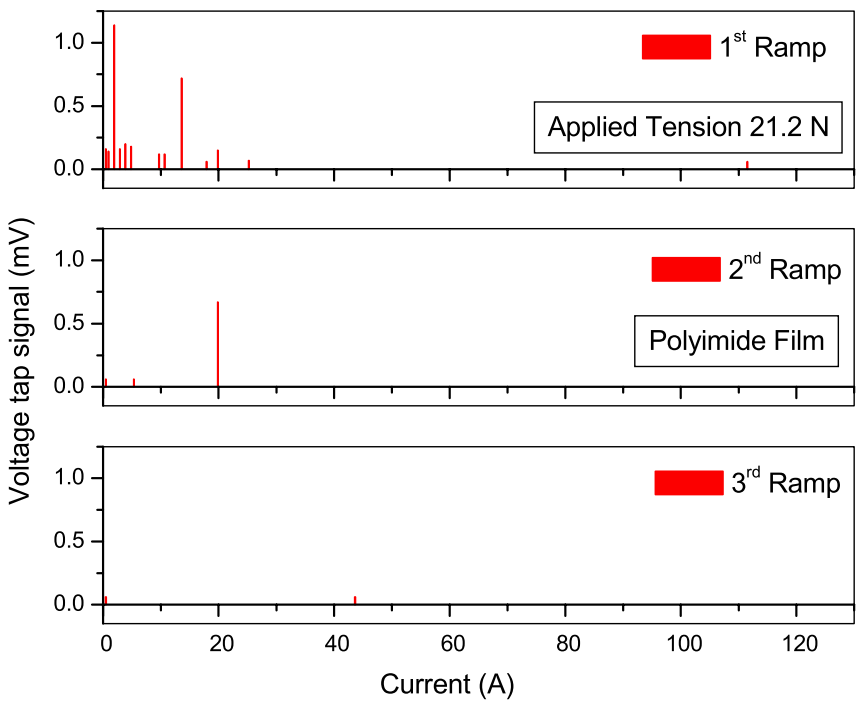

FIG. 3. (Color) Training effect as observed in the case of polyimide film. 


\section{B. Experimental findings \\ 1. Polyimide film}

Large wire motions were observed when the wire tension was $7.1 \mathrm{~N}$. The amount of frictional heat generated during these wire motions was sufficient to quench the wire. Figure 2 shows the amplitude of the voltage tap signal measured using the pen recorder with the wire tension set at $14.1 \mathrm{~N}$. Figure 3 shows the training behavior when the tension is $21.2 \mathrm{~N}$. At higher tension the voltage spike amplitude decreased. The number of voltage spikes also reduced from ramp to ramp.

\section{Dyneema cloth}

A large number of voltage spikes with small amplitude were observed when the tension was $7.1 \mathrm{~N}$. Heat generated during these wire motions was not sufficient to quench the

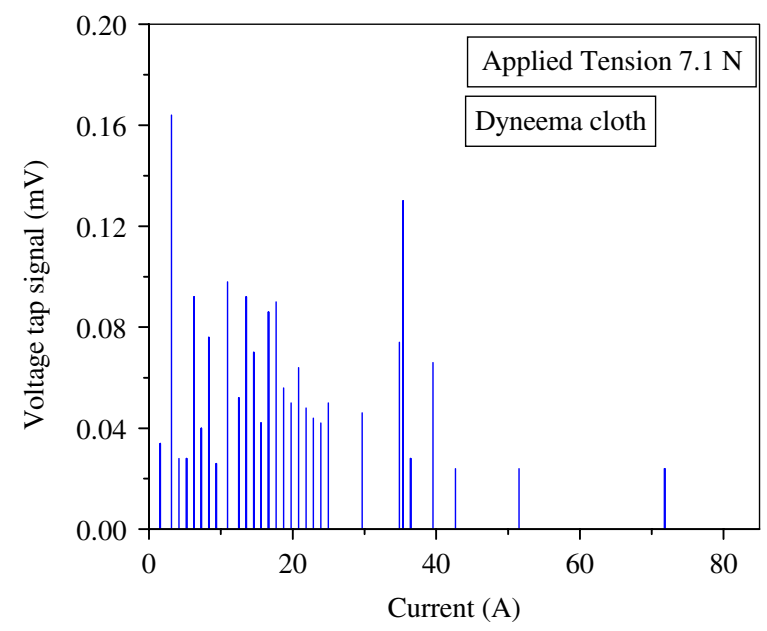

FIG. 4. (Color) Voltage tap signal as a function of current.

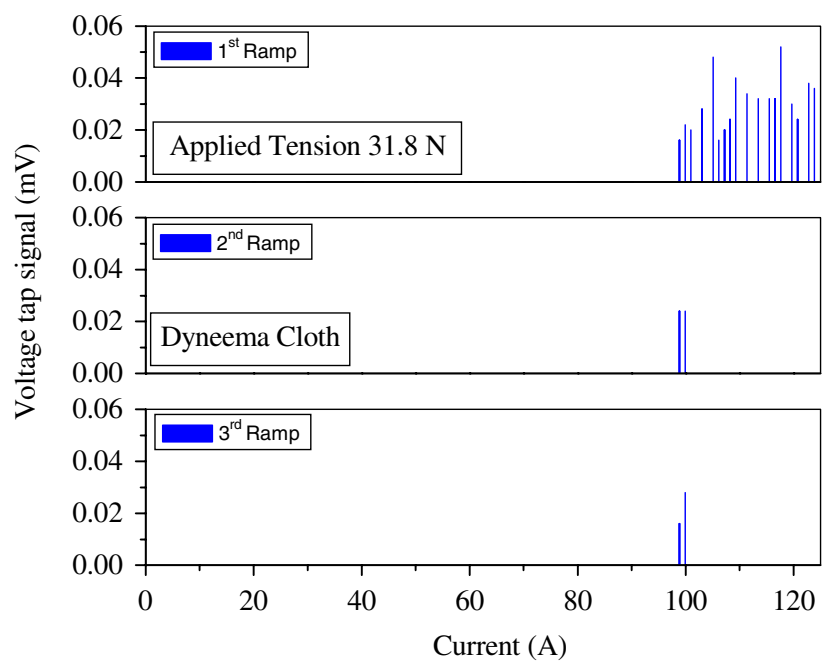

FIG. 5. (Color) Training effect as observed in the case of Dyneema cloth.

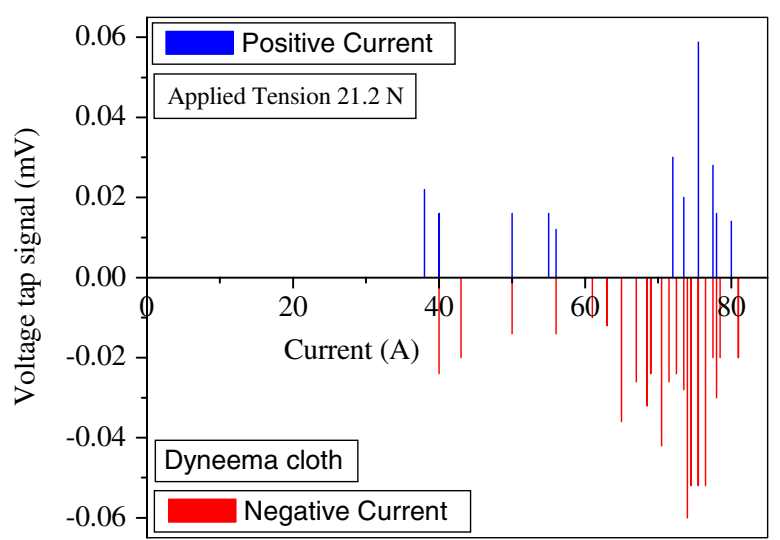

FIG. 6. (Color) Comparison of voltage tap signal after reversing the current polarity.

wire. The voltage tap signal measured using the pen recorder is shown in Fig. 4. Figure 5 shows the training behavior when the wire tension was $31.8 \mathrm{~N}$. At higher tension the voltage spike amplitude decreased and a larger electromagnetic force was needed to start the wire motion.

Reversing the polarity of the current in the superconducting wire erased the history and no significant effect on the electromagnetic force needed to start the wire motion was observed. However, an asymmetric voltage signal pattern was observed, presumably due to the asymmetric position of superconducting wire in the head part. A typical pattern obtained when the wire tension was $21.2 \mathrm{~N}$ is shown in Fig. 6.

\section{Comparison between polyimide film and Dyneema cloth}

Figure 7 shows the current at which the wire motion starts as a function of tension for both the polyimide film and the Dyneema cloth. At larger tension values the level

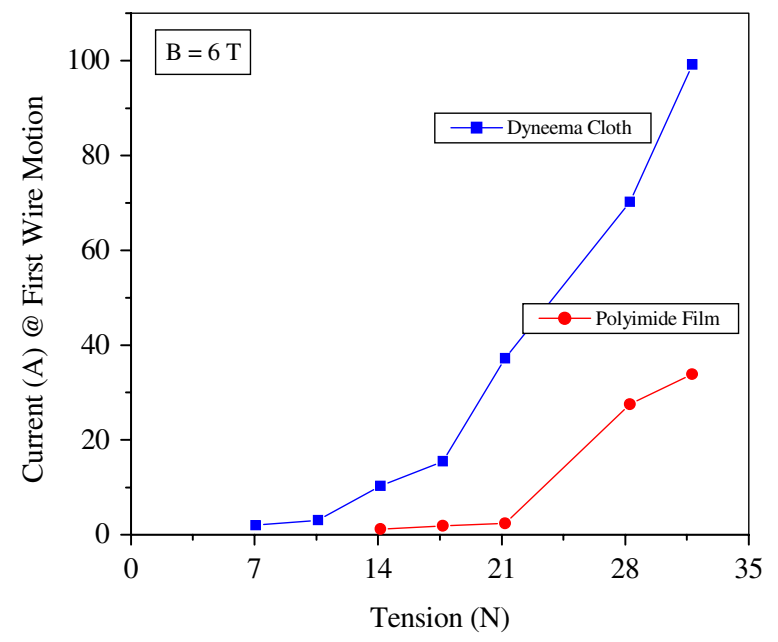

FIG. 7. (Color) Dependence of current at which wire motion starts with tension. 

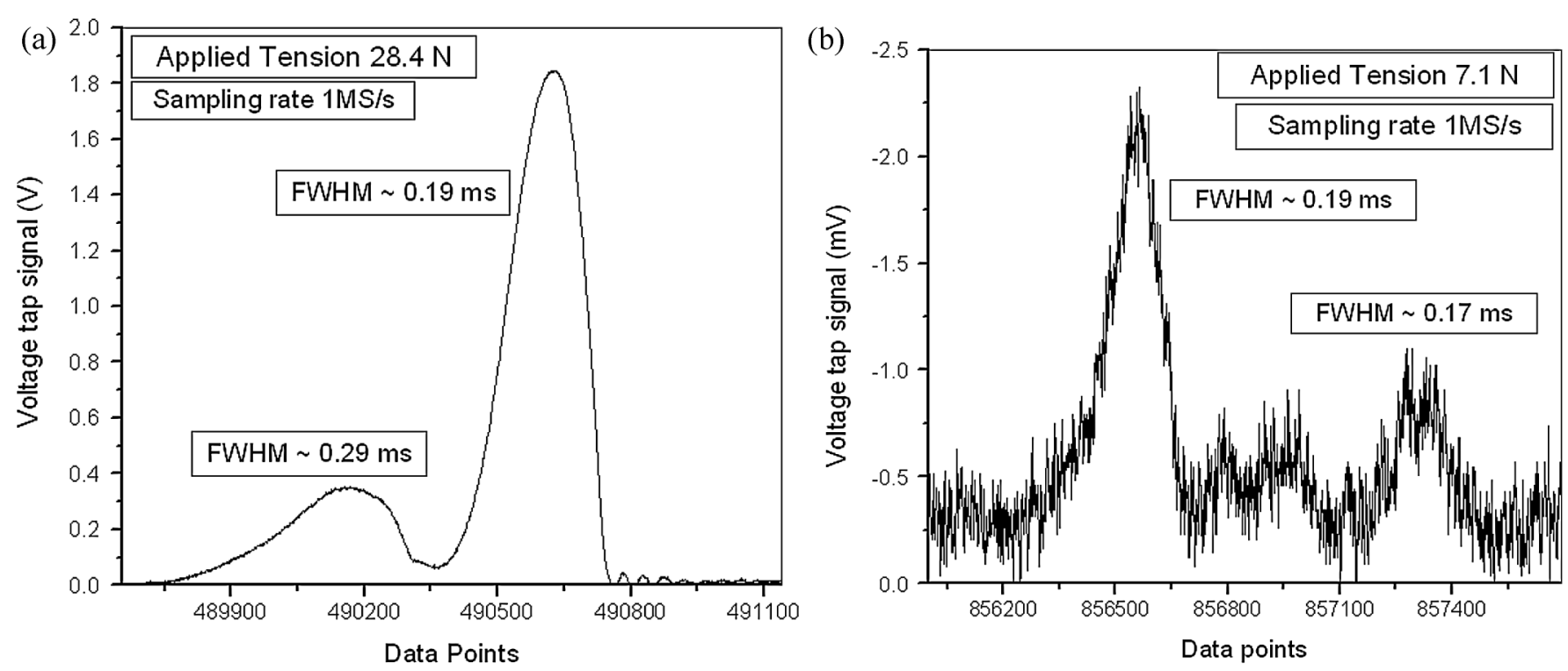

FIG. 8. (a) Voltage spike in the case of polyimide film. (b) Voltage spike in the case of Dyneema cloth.

of electromagnetic force needed to start the wire motion increases for both materials. One of the speculations for larger electromagnetic force in the case of Dyneema cloth as compared to polyimide film is the coarser texture of the cloth. The superconducting wire might have become embedded in the Dyneema cloth.

The pen recorder was not suitable for measurements of short duration pulses. So, we used a commercially available, 16-bit data recorder to measure the voltage tap signal. The sampling rate of the recorder was $1 \mathrm{MS} / \mathrm{s}$. Figure 8(a) shows the typical voltage spike pattern when the applied tension is $28.4 \mathrm{~N}$ and the base insulating material is polyimide film. The full width at half maximum (FWHM) for the main peak is $\sim 0.19 \mathrm{~ms}$. Figure 8 (b) shows the typical voltage spike pattern when the applied tension is $7.1 \mathrm{~N}$ and the base insulating material is Dyneema cloth. The FWHM for the main peak is $\sim 0.19 \mathrm{~ms}$.

The voltage tap signal amplitude measured by the data recorder was more than 1 order of magnitude greater than with the pen recorder. The difference in voltage tap signal amplitude was validated by feeding a short duration pulse generated using a pulse generator to the data recorder and the pen recorder.

\section{CONCLUSION}

In this study, an experimental setup to study superconducting wire motion under the influence of electromagnetic force has been developed and we have verified the effectiveness of this system. The amplitude of voltage signal generated due to wire motion is more than 2 orders of magnitude less in the case of Dyneema cloth as compared to polyimide film. Hence, the use of Dyneema cloth may reduce the frictional heat generated due to wire motion and could make magnet performance more reliable. At higher levels of applied tension the voltage spike amplitude decreased and larger electromagnetic force was needed to start wire motion.

The experimental results are repeatable under the same experimental conditions and no significant dependence of the voltage spike FWHM on wire current ramp rate or applied tension was observed. The FWHM is of the same order for both samples. No significant difference in peak amplitude was observed on reversing the polarity of current in the superconducting wire.

In the next study, we will perform experiments using sheet material fabricated with Dyneema fiber. In this way we aim to overcome the embedding of the superconducting wire in Dyneema cloth.

\section{ACKNOWLEDGMENTS}

The authors would like to thank Mr. Yuuji Kojima for his help in conducting the experiments.

[1] N. Yanagi, K. Seo, S. Imagawa, H. Sekiguchi, K. Takahata, S. Yamada, T. Mito, T. Ishigohka, and A. Ninomiya, Fusion Eng. Des. 81, 2561 (2006).

[2] A. Devred, The Physics of Particle Accelerators, AIP Conf. Proc. No. 1262 (AIP, New York, 1990).

[3] T. Ogitsu, K. Tsuchiya, and A. Devred, IEEE Trans. Magn. 27, 2132 (1991).

[4] R. S. Kensley and Y. Iwasa, Cryogenics 20, 25 (1980).

[5] A. Yamanaka, T. Kashima, S. Nago, K. Hosoyama, T. Takao, S. Sato, and M. Takeo, Physica (Amsterdam) 372C-376C, 1447 (2002). 
[6] T. Kashima, A. Yamanaka, S. Takasugi, and S. Nishihara, Adv. Cryog. Eng. 46, 329 (2000).

[7] T. Takao, T. Nemoto, H. Konda, T. Kashima, and A. Yamanaka, ICMC Report No. ICECE16/ICMC (1997), p. 1993.
[8] K. Ruwali, A. Yamanaka, Y. Teramoto, K. Nakanishi, and K. Hosoyama, in Proceedings of the 11th European Particle Accelerator Conference, Genoa, 2008 (EPS-AG, Genoa, Italy, 2008), p. 2449. 